\title{
Effect of rosiglitazone on insulin resistance, C-reactive protein and endothelial function in non-obese young women with polycystic ovary syndrome
}

\author{
İlhan Tarkun, Berrin Çetinarslan, Erdem Türemen, Tayfun Şahin ${ }^{1}$, Zeynep Cantürk and Baki Komsuoğlu ${ }^{1}$ \\ Endocrinology and Metabolism Department, and ${ }^{1}$ Cardiology Department, Kocaeli University, Turkey
}

(Correspondence should be addressed to İ Tarkun, Yahyakaptan Mah. A-19 Blok D.17, 41050, Bekirpaşa-Kocaeli, Turkey; Email: ilhantarkun@superonline.com)

\begin{abstract}
Objective: Women with polycystic ovary syndrome (PCOS) exhibit elevated levels of serum C-reactive protein (CRP) and impaired endothelium dysfunction which are directly correlated with insulin resistance. Because rosiglitazone improves insulin sensitivity, we tested whether rosiglitazone treatment ameliorates high-sensitivity (hs)CRP levels and endothelial dysfunction in these patients.

Design: Thirty-one women with PCOS were recruited (mean age, $24.7 \pm 3.9$ (s.E.) years; mean body mass index (BMI), $25.6 \pm 3.2 \mathrm{~kg} / \mathrm{m}^{2}$ ). All women were treated with $4 \mathrm{mg}$ rosiglitazone daily for 12 months.

Methods: Serum levels of testosterone, LH, FSH, sex hormone-binding globulin (SHBG), insulin and hsCRP were measured. The BMI, hirsutism scores and insulin sensitivity indices were calculated before and after treatment. Arterial endothelium and smooth muscle function was measured by examining brachial artery responses to endothelium-dependent and endothelium-independent stimuli before and after treatment.

Results: After treatment with rosigitazone there were significant decreases in serum testosterone $(91.2 \pm 37.5$ vs $56.1 \pm 21.8 \mathrm{ng} / \mathrm{dl} ; P<0.01)$ and fasting insulin concentrations $(12.5 \pm 7.6$ vs $8.75 \pm 4.03 \mu \mathrm{U} / \mathrm{ml} ; P=0.015)$. Insulin resistance indices were significantly improved after rosiglitazone treatment $(P<0.05)$. There were no significant changes in BMI, waist circumference, serum total cholesterol, low-density lipoprotein (LDL)-cholesterol, FSH and LH levels. Hirsutism score was decreased significantly after treatment $(10.8 \pm 1.8$ vs $7.6 \pm 1.7 ; P<0.05)$. Twenty-four of the women reverted to regular menstrual cycles. Levels of SHBG increased significantly after treatment $(28.7 \pm 8.7$ vs $48.4 \pm 11.2 \mathrm{nmol} / \mathrm{l} ; P<0.01)$. Serum hsCRP levels were decreased significantly after rosiglitazone treatment $(0.25 \pm 0.1$ vs $0.09 \pm 0.02 \mathrm{mg} / \mathrm{dl} ; P=0.006)$. There was also significant improvement in endothelium-dependent vascular responses after rosiglitazone treatment $(9.9 \pm 3.9$ vs $16.4 \pm 5.1 \% ; P<0.01)$.

Conclusions: We conclude that rosiglitazone treatment improves insulin sensitivity in women with PCOS. It also decreases androgen production without significant weight gain. More importantly, it has beneficial effects on endothelial dysfunction and low-grade chronic inflammation in normal weight young women with PCOS.
\end{abstract}

European Journal of Endocrinology 153 115-121

\section{Introduction}

Polycystic ovary syndrome (PCOS) is characterized by hyperandrogenism and chronic anovulation but insulin resistance and hyperinsulinism play a critical role in the syndrome pathogenesis $(1,2)$. Women with PCOS have a clustering of cardiovascular risk factors other than hyperinsulinemia such as obesity, lipid abnormalities, hypertension and changes in fibrinolytic system $(3,4)$. Over the past 15 years, large epidemiological studies describing cardiovascular disease risk factors among women with PCOS have been carried out (5-8). These studies demonstrated that PCOS women exhibit significantly adverse coronary heart disease (CHD) risk profiles at relatively young age (8), suggesting that these increases may result in premature coronary atherosclerosis. Given the high prevalance of PCOS in the female population, this condition may potentially account for a significant proportion of atherosclerotic heart disease among younger women.

Because CHD is characterized by a long incubation period, metabolic abnormalities observed in the teens 
or twenties among PCOS women might translate into measurable vascular abnormalities by middle age. Endothelial dysfunction has been regarded as an early feature of atherosclerosis and plays an important role in the development of atherosclerotic diseases $(9,10)$. Dysfunction of endothelium cells is probably the earliest event in the process of lesion formation (11). Measures of endothelial dysfunction, such as brachial artery ultrasound, allow for the identification, in a non-invasive manner, of subjects at risk for cardiovascular disease early in its course so that risk-factor modification can be instituted to delay or prevent cardiovascular events. Assessment of endothelial function by measuring flow-mediated dilatation (FMD) of the brachial artery is currently being regarded as a potential tool for predicting CHD (12).

Studies showing a link between endothelial dysfunction and insulin resistance have been reported (13, 14). Endothelial dysfunction might therefore contribute to the increased risk of atherosclerosis in insulin-resistant subjects, such as those with PCOS (15). Accumulating evidence suggests that atherosclerosis represents a chronic inflammatory process and inflammatory markers like C-reactive protein (CRP) provide an adjunctive method for global assessment of cardiovascular risk (16). Previous studies also detected a correlation between insulin resistance and CRP concentrations (17-19). Endothelial dysfunction and high concentrations of high-sensitivity (hs)CRP were detected even in normal-weight women with PCOS (15). Clinical strategies aimed at reducing insulin resistance may prevent early atherosclerosis in women with PCOS. Recently, insulin sensitizers have been used to reduce the level of hyperinsulinemia and its negative impact on ovarian function and possibly to prevent long-term consequences of hyperinsulinemia. Thiazolidinediones (TZDs) are oral anti-diabetic agents that exert their insulin-sensitizing actions through the peroxisome-activated receptor; they are found in a number of tissues including liver, skeletal muscle and adipose tissue. Troglitazone, a member of the TZD family, was found to have beneficial effects on insulin sensitivity and ovarian function in women with PCOS (20-22). In a previous study, endothelial dysfunction in women with PCOS was improved by troglitazone therapy (23). However, troglitazone was taken off the market as a result of concerns over its hepatotoxicity. A newer member of the TZD family, rosiglitazone, was found to have beneficial effects on insulin sensitivity and endothelial dysfunction in both diabetic and nondiabetic patients (24-26). Rosiglitazone may affect inflammatory pathways through transcriptional mechanisms. These anti-inflammatory effects raise the prospect of reduced cardiovascular risk. Currently there are only limited data on the use of rosiglitazone in PCOS.

The aim of our present study is to evaluate the efficiency of rosiglitazone therapy on insulin resistance, serum concentrations of pro-inflammatory markers like
hsCRP and endothelial dysfunction in young women with PCOS.

\section{Materials and methods}

Thirty-one women affected by PCOS (mean age, $24.7 \pm 3.9$ years) were enrolled in our study. The study protocol was approved by the Ethics Committee for Human Studies of Kocaeli University Hospital. Informed, written consent was obtained from all subjects after explanation of the nature, purpose and potential risks of the study. PCOS was defined when at least two of the following three features were present after the exclusion of other etiologies (Rotterdam criteria) (27): oligo- or anovulation (fewer than six menstrual periods in the preceding year); clinical (Ferriman-Gallwey score > 8) (28) and/or biochemical signs of hyperandrogenism and polycystic ovaries. Biochemical criteria included an abnormal luteinizing hormone (LH):follicle-stimulating hormone $(\mathrm{FSH})$ ratio $(>2)$ and/or elevated testosterone levels. The ultrasound criteria used for diagnosis of PCO were: presence of 12 or more follicles in each ovary measuring 2-9 $\mathrm{mm}$ in diameter, and/or increased ovarian volume $(>10 \mathrm{ml})$. Oligomenorrhea or amenorrhea were present in 20 of the 31 patients (mean cycle length, 52.7 \pm 12.8 days). All women had normal thyroid, renal and hepatic functions. Their prolactin levels were within normal limits. Exclusion criteria for all subjects included: pregnancy or planning pregnancy within a year; current or previous use (within 6 months) of oral contraceptives, anti-androgens, anti-diabetics, statins, glucocorticoids or other hormonal drugs; cigarette smoking; chronic alcohol consumption; blood pressure of $\geq 130 / 85 \mathrm{mmHg}$ or treated hypertension; known cardiovascular disease (CVD); hypertension; diabetes mellitus. An overnight dexamethazone suppression test (1 mg) and follicular phase serum 17-OH progesterone determination were performed in order to exclude Cushing's syndrome and late-onset congenital adrenal hyperplasia.

All blood samples were obtained in the morning between 0800 and $0900 \mathrm{~h}$ after a 3-day, $300 \mathrm{~g}$ carbohydrate diet, an overnight fasting and during early follicular phase. During the same visit all subjects underwent anthropometric measurements, an oral glucose tolerance test and transvaginal ultrasonography. The serum concentrations of FSH, LH, testosterone, prolactin and sex hormone-binding globulin (SHBG) were measured by chemiluminescent enzyme immunoassay (Immulite 2000; Diagnostic Products Corporation, Los Angeles, CA, USA) Serum glucose was measured by using a glucokinase technique. Lipid analysis in fasting serum was performed for all patients. The lipid profile included measurement of the levels of total cholesterol, high- and low-density lipoprotein (HDL and LDL)-cholesterol and triglyceride. These parameters were measured by commercial enzymatic 
methods (Aeroset automated analyzer, Abbott Diagnostics, IL, USA). LDL-cholesterol was calculated using Friedewald's formula.

Plasma insulin levels were measured by chemiluminescent enzyme immunoassay (Immulite 1000 Analyser) with inter-assay and intra-assay coefficients of variation $(\mathrm{CV})$ that did not exceed $6.4 \%$. Plasma hsCRP concentrations were measured by chemiluminescent enzyme immunoassay (Immulite 2000) with intra-assay CV of $8.7 \%$ and a sensitivity of $0.01 \mathrm{mg} / \mathrm{dl}$ . The upper limit of detection was $15 \mathrm{mg} / \mathrm{dl}$.

Insulin resistance was determined by a number of different methods including fasting insulin, the homeostasis model assessment (HOMA) and the quantitative insulin sensitivity check index (QUICKI). The estimate of insulin resistance by HOMA score was calculated using the formula: fasting serum insulin $(\mu \mathrm{U} / \mathrm{ml}) \times$ fasting plasma glucose $(\mathrm{mmol} / \mathrm{l}) / 22.5$ (29). The QUICKI is derived by calculating the inverse of the sum of logarithmically expressed values of fasting insulin and glucose (30).

Using ultrasonography, arterial endothelium and smooth muscle function were measured by examining brachial artery responses to endothelium-dependent (FMD) and endothelium-independent stimuli (sublingual nitroglycerin (NTG)). Ultrasonographic measurements were carried out according to the method described by Coretti et al. (31). The assessment was performed after an overnight fast in a quiet, air-conditioned room $\left(22-24^{\circ} \mathrm{C}\right)$ by one experienced cardiologist who was blinded to diagnosis. The diameter of the brachial artery was measured on B-mode ultrasound images, using a $7.5 \mathrm{MHz}$ transducer. Ultrasound measurements were obtained using a high-resolution ultrasound machine (Toshiba Power Vision 8000). The right brachial artery was scanned in longitudinal sections $2-8 \mathrm{~cm}$ above the elbow. After the detection of the right transducer position, the skin surface was marked and the arm kept in the same position during the study. All scans were recorded on videorecorder and analyzed later. Arterial diameters were measured at rest, during reactive hyperemia (FMD), again at rest and after administration of $0.4 \mathrm{mg}$ sublingual NTG. Reactive hyperemia was induced by inflation of a pneumatic cuff on the upper arm to suprasystolic pressure, followed by cuff deflation after $4.5 \mathrm{~min}$. The diameter of the brachial artery was scanned and recorded after deflation. After $10-15 \mathrm{~min}$ rest, the second control scan diameter was recorded. Then sublingual NTG was administered and 3.5-4 min later a final scan of the diameter was recorded.

The end-diastolic arterial diameter was measured from one media-adventitia interface to the other at the clearest section; measurements were taken 3 times at baseline, every $20 \mathrm{~s}$ after reactive hyperemia and after administration of NTG. The maximum vessel diameter was defined as the average of the three consecutive maximum diameter measurements after hyperemia and NTG respectively. Vasodilatation by reactive hyperemia or NTG was expressed as the percentage change in diameter compared with baseline values. The intra-observer variation in our clinic was $1.5 \%$.

All of the women were treated with rosiglitazone, $4 \mathrm{mg}$ once a day. Patients were seen every 8 weeks for control of compliance and assessment of side effects. After 12 months of the treatment, the women were admitted to the clinical research center and all of the clinical examinations and laboratory evaluations were repeated. All of the results obtained were then analyzed and pre- and post-treatment values were compared.

\section{Statistical analysis}

The Statistical Package for the Social Sciences (SPSS version 11.5 for Windows) was used for statistical analysis. The person carrying out the data analysis was blinded to diagnosis. Results are expressed as means \pm s.D. The characteristics of distribution were tested using the Kolmogorov-Smirnof test. Highly skewed variables were analysed after logarithmic transformation. Spearman's rank correlations were used for these variables. The Mann-Whitney U-test was used for variables with persisting skewed distribution after log transformation. Differences between means were analyzed using Student's unpaired t-test using two-tailed tests for significance. $P<0.05$ was considered statistically significant. Analysis of correlations between parameters was performed using Pearson's bivariate correlation coefficient.

\section{Results}

All of the 31 women who had enrolled completed the study. At the dosage used there were no adverse effects reported by our subjects. During the treatment period no elevations in serum transaminases were detected. Twenty-four of the 31 patients studied reverted to regular ovulatory cycles during the treatment. The length of the cycles at the end of the treatment was 33.7 \pm 6.7 days. Clinical characteristics and hormonal parameters of the patients are shown in Table 1. In our study rosiglitazone therapy caused a small $(0.8 \mathrm{~kg})$ but non-significant increase in body weight without changes in waist circumferences. There was no significant change in total cholesterol, LDL, LH and FSH levels. The hirsutism score decreased significantly after rosiglitazone treatment $(10.8 \pm 1.8$ vs $7.6 \pm 1.7 ; P<0.05)$. Serum triglyceride concentrations were decreased and serum HDL concentrations were increased after treatment but these changes were statistically insignificant $(P>0.05)$. Serum testosterone levels decreased significantly after rosiglitazone treatment (91.2 \pm 37.5 vs $56.1 \pm 21.8 \mathrm{ng} / \mathrm{dl} ; \quad P<0.01)$ where as SHBG levels increased significantly $(28.7 \pm 8.7 \quad$ vs $48.4 \pm 11.2 \mathrm{nmol} / \mathrm{l} ; \quad P<0.01)$. Serum hsCRP levels 
Table 1 Patient characteristics and hormone studies before and after rosiglitazone treatment. Values expressed as means \pm S.E.

\begin{tabular}{lccc}
\hline & Before rosiglitazone $(n=31)$ & Post-rosiglitazone $(n=31)$ & $P$ \\
\hline BMI $\left(\mathrm{kg} / \mathrm{m}^{2}\right)$ & $25.6 \pm 3.2$ & $26.3 \pm 3.2$ & 0.375 \\
Waist circumference $(\mathrm{cm})$ & $81.25 \pm 8.04$ & $81.22 \pm 8.6$ & 0.448 \\
Ferriman-Gallwey score & $10.8 \pm 1.8$ & $7.6 \pm 1.7$ & 0.02 \\
LH $(\mathrm{IU} / \mathrm{l})$ & $7.9 \pm 8.4$ & $8.2 \pm 7.3$ & 0.533 \\
FSH $(\mathrm{IU} / \mathrm{l})$ & $4.89 \pm 2.14$ & $5.9 \pm 2.5$ & 0.521 \\
Testosterone $(\mathrm{ng} / \mathrm{dl})$ & $91.2 \pm 37.5$ & $56.1 \pm 21.8$ & $<0.01$ \\
SHBG $(\mathrm{nmol} / \mathrm{l})$ & $28.7 \pm 8.7$ & $48.4 \pm 11.2$ & $<0.01$ \\
Total cholesterol $(\mathrm{mg} / \mathrm{dl})$ & $163.2 \pm 36.8$ & $160.1 \pm 38.9$ & 0.456 \\
Triglyceride $(\mathrm{mg} / \mathrm{dl})$ & $121 \pm 109.48$ & $114.1 \pm 75.7$ & 0.095 \\
HDL-cholesterol $(\mathrm{mg} / \mathrm{dl})$ & $46.1 \pm 11.9$ & $50.4 \pm 13.05$ & 0.112 \\
LDL-cholesterol $(\mathrm{mg} / \mathrm{dl})$ & $96.41 \pm 23.2$ & $100.4 \pm 25.5$ & 0.319 \\
& & &
\end{tabular}

decreased significantly after treatment (Fig. 1). Glucose metabolism parameters of the patients are shown in Table 2. There was no significant change in fasting serum glucose concentrations. However, fasting insulin concentrations and insulin sensitivity indices (HOMA and QUICKI) were changed significantly $(P=<0.01)$ after rosiglitazone treatment.

There was a significant improvement in endothelium-dependent (FMD) vascular responses after rosiglitazone treatment $(9.9 \pm 3.9$ vs $16.4 \pm 5.1 \%$; $P<0.01)$. Baseline artery diameter and endotheliumindependent vascular responses were not changed with treatment (Table 3). At baseline, endotheliumdependent vasodilatation was significantly correlated with insulin resistance and hsCRP levels (Pearson's correlation, $P<0.05)$.

\section{Discussion}

The endothelium is highly active metabolically and plays a key role in vascular homeostasis through the release of a variety of autocrine and paracrine substances. The healthy endothelium, particularly endothelium-derived nitric oxide (NO), not only modulates the tone of underlying vascular smooth muscle but also inhibits several proatherogenic processes,

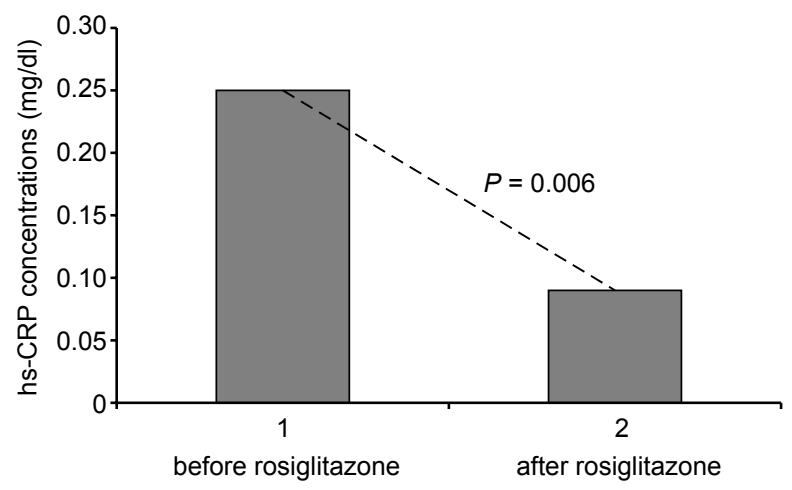

Figure 1 hs-CRP concentrations in women with PCOS before and after rosiglitazone treatment. including monocyte and platelet adhesion, oxidation of LDLs, synthesis of inflammatory cytokines, smooth muscle proliferation and migration, and platelet aggregation; thus exhibiting important anti-atherogenic effects (32). Endothelial dysfunction has been regarded as an early feature of atherosclerosis and plays an important role in the development of atherosclerotic disease $(9,33)$. Assessment of endothelial function by different methods has emerged as a tool for detection of evidence of preclinical cardiovascular disease (34). Brachial artery ultrasound is a widely used non-invasive measure of endothelial cell function. The forearm blood flow is occluded using a blood pressure cuff maintained at a standard pressure. When pressure is released, reactive hyperemia occurs. This results in shear-stress-induced NO release and subsequent vasodilatation (FMD). This technique has the advantage of being non-invasive and can readily identify populations with attenuated endothelial function. It is particularly well suited for the study of the earliest stages of atherosclerosis in children and young adults, thus providing maximal opportunity for prevention (31). Endothelium-independent stimuli such as nitroprusside or glyceryl trinitrate, which act as NO donors, are used to assess whether the observed findings are secondary to NO-mediated effects or the results of abnormalities of the vascular smooth muscle cells.

Reduced endothelium-dependent vasodilatation has previously been reported in various clinical studies investigating disorders associated with increased risk of CHD, such as hypertension (35) and hypercholesterolemia (36); and more recently insulin resistance (37, 38). Previous studies have demonstrated that secondary hyperinsulinemia is the key factor responsible for the hyperandrogenism characteristic of PCOS, which is attributed to increased stimulation of the activity of the cytochrome P450c $17 \alpha$ in the ovary $(39,40)$. Hyperinsulinemia also decreases the circulating concentrations of SHBG and contributes to greater concentrations of free androgens in the blood (41). Both lean and obese women with PCOS have peripheral insulin resistance and hyperinsulinemia that appear to play a pathogenic role in the disease $(15,42,43)$. Previous 
Table 2 Glucose metabolism parameters of the patients before and after rosiglitazone therapy. Values expressed as means \pm S.E.

\begin{tabular}{lccr}
\hline & Before rosiglitazone $(n=31)$ & Post-rosiglitazone $(n=31)$ & $\boldsymbol{P}$ \\
\hline Fasting glucose $(\mathrm{mg} / \mathrm{dl})$ & $91.4 \pm 9.1$ & $87.1 \pm 7.65$ & 0.234 \\
Fasting insulin $(\mu \mathrm{U} / \mathrm{ml})$ & $12.5 \pm 7.6$ & $8.75 \pm 4.03$ & 0.015 \\
HOMA & $2.94 \pm 1.8$ & $1.97 \pm 0.8$ & $<0.01$ \\
QUICKI & $0.34 \pm 0.02$ & $0.381 \pm 0.005$ & $<0.01$ \\
\hline
\end{tabular}

studies have shown an endothelial dysfunction in women with PCOS $(15,44,45)$. Accumulating evidence also suggests that atherosclerosis represents a chronic inflammatory process and inflammatory markers like CRP provide an adjunctive method for global assessment of cardiovascular risk (46, 47). Recent data also suggest that CRP may directly promote endothelial dysfunction by increasing the synthesis of soluble adhesion molecules, increasing monocyte chemoattractant protein secretion and facilitating macrophage LDL uptake (48). In previous studies, high hsCRP levels and a link between insulin resistance and elevated CRP levels was shown in women with $\operatorname{PCOS}(15,26,49)$.

Evidence for a causal role of insulin in the ovarian abnormalities of PCOS is derived from the clinical use of insulin-sensitizing agents. Insulin-sensitizing interventions directly targeting pathogenetic causes of the PCOS may also have beneficial effects on the levels of markers for cardiovascular disease. Since endothelial dysfunction and low-grade chronic inflammation are considered to be an early and necessary abnormality in the pathogenesis of atherosclerosis, we examined the effect of insulin-sensitizing agent rosiglitazone on hsCRP levels and endothelial dysfunction in young women with PCOS. Troglitazone, a peroxisome proliferator-activated receptor $\delta$ agonist that has been taken off the market, has been shown to be effective in improving endocrine and ovulatory performance in women with PCOS $(21,22)$. Previous studies on the effect of rosiglitazone in women with PCOS are limited. Recently, Sepilian et al. (50) reported that rosigitazone treatment improves ovulatory function and decreases insulin resistance in women with PCOS. We also detected decreased insulin resistance and androgen levels and also improvement of ovulatory cycles in women with PCOS after rosiglitazone treatment. In a recent study, Baillargeon et al. (51) found that insulin-sensitizing drugs increase ovulatory frequency and ameliorate hyperinsulinemia even in non-obese women with PCOS who appear to have normal insulin sensitivity. They suggested that some women with PCOS may have a primary ovarian hyper-responsiveness to insulin without global insulin resistance. Paradisi et al. (23) showed that troglitazone therapy improves endothelial dysfunction in women with PCOS. Pistrosch et al. (24) showed that in type 2 diabetes rosiglitazone therapy for insulin resistance ameliorates endothelial dysfunction independent of glucose control (24). The effects of rosiglitazone in improving endothelial function were associated with improvement in NO generation (52). Rosiglitazone improves endothelial dysfunction and decrease hsCRP levels in non-diabetic patients with coronary artery disease (25) and also in non-diabetic patients with metabolic syndrome (26). Rosiglitazone exerts a potent anti-inflammatory effect at the cellular and molecular level and in plasma (53). We found that rosiglitazone treatment improves endothelium-dependent vasodilatation and decreases serum levels of pro-inflammatory marker hsCRP levels in young women with PCOS. Haffner et al. (54) showed that rosiglitazone reduces serum levels of pro-inflammatory marker CRP in patients with type 2 diabetes. The present study is the first to our knowledge to demonstrate beneficial effects of rosiglitazone treatment on endothelial dysfunction and hsCRP levels in non-obese young women with PCOS.

In conclusion, our results indicate that an insulinsensitizing drug, rosiglitazone, improves insulin sensitivity in women with PCOS. It also helps to restore ovulation and decrease androgen production without significant weight gain and other side effects. More importantly, these data indicate for the first time that clinical strategies aimed at reducing insulin resistance may have a cardioprotective effect, even in non-obese women with PCOS. Further prospective and placebo controlled studies with larger numbers of patients and long-term follow-up are necessary to determine whether insulin-sensitizing therapy can be useful in modifying cardiovascular risk in women with PCOS.

Table 3 Brachial artery responses, expressed as percentage dilatation from baseline.

\begin{tabular}{lccc}
\hline & Before rosiglitazone $(n=31)$ & Post-rosiglitazone $(n=31)$ & $\boldsymbol{P}$ \\
\hline Baseline artery diameter $(\mathrm{mm})$ & $3.35 \pm 0.27$ & $3.34 \pm 0.34$ & 0.815 \\
FMD $(\%)$ & $9.9 \pm 3.9$ & $16.4 \pm 5.1$ & $<0.01$ \\
NTG $(\%)$ & $19.1 \pm 5.2$ & $21.1 \pm 6.3$ & 0.218 \\
\hline
\end{tabular}




\section{References}

1 Franks S. Polycystic ovary syndrome. New England Journal of Medicine $1995333853-861$.

2 Nestler JE. Role of hyperinsulinemia in the pathogenesis of polycystic ovary syndrome, and its clinical implications. Seminars in Reproductive Endocrinology 199715 111-122.

3 Talbott EO, Guzick DS, Clerici A, Berga S, Detre K, Weimer K \& Kuller H. Coronary heart disease risk factors in women with polycystic ovary syndrome. Arteriosclerosis Thrombosis and Vascular Biology 199515 821-826.

4 Tarkun İ, Cantürk Z, Arslan BÇ, Türemen E \& Tarkun P. The plasminogen activator system in young and lean women with polycystic ovary syndrome. Endocrine Journal 200451 467-472.

5 Dahlgreen E, Janson PO, Johansson S, Lapidus L \& Oden A. Polycystic ovary syndrome and risk of myocardial infarction evaluated from a risk factor model based on a prospective population study of women. Acta Obstetricia et Gynecologica Scandinavica 1992 $71599-604$.

6 Conway GS, Agrawal R, Betteridge DJ \& Jacobs HS. Risk factors for coronary artery disease in lean and obese women with polycystic ovary syndrome. Clinical Endocrinology 199237 119-125.

7 Dahlgreen E, Johansson S, Lindstedt G, Knutsson F, Oden A, Janson PO, Mattson LA, Crona N \& Lundberg PA. Women with polycystic ovary syndrome resected in 1956 to 1965: a longterm follow-up focusing on natural history and circulating hormones. Fertility and Sterility 199257 505-513.

8 Talbott E, Clerici A, Berge SL, Kuller L, Guzick D, Detre K, Daniels T \& Engberg RA. Adverse lipid and coronary heart disease risk profiles in young women with polycystic ovary syndrome: results of a case-control study. Journal of Clinical Epidemiology 1998 $51415-422$.

9 Ross R. Atherosclerosis: an inflammatory disease. New England Journal of Medicine 1999340 115-126.

10 Shimokawa H. Primary endothelial dysfunction: atherosclerosis. Journal of Molecular and Cellular Cardiology 199931 23-27.

11 Verma S, Buchanan MR \& Anderson TJ. Endothelial function testing as a biomarker of vascular disease. Circulation 2003 $1082054-2059$

12 Anderson TJ, Uehata A, Gerhard MD, Meredith IT, Knab S, Delagrange D, Lieberman EH, Ganz P, Creager MA \& Yeung AC. Close relation of endothelial function in human coronary and peripheral circulations. Journal of American Collage of Cardiology 1995 26 1235-1241.

13 Arcaro G, Zamboni M, Rossi L, Turcato E, Covi G, Armellini F, Basella O \& Lechi A. Body fat distribution predicts the degree of endothelial dysfunction in uncomplicated obesity. International Journal of Obesity 199923 936-942.

14 Steinberg HO, Chaker H, Leaming R, Johnson A, Brechtel G \& Boron AD. Obesity/insulin resistance is associated with endothelial dysfunction. Implications for the syndrome of insulin resistance. Journal of Clinical Investigations 199611 2601-2610.

15 Tarkun İ, Arslan BÇ, Cantürk Z, Türemen E, Şahin T \& Duman C. Endothelial dysfunction in young women with PCOS: relationship with insulin resistance and low-grade chronic inflammation. Journal of Clinical Endocrinology and Metabolism $2004895592-5596$.

16 Verma $S \&$ Anderson TJ. Fundamentals of endothelial function for the clinical cardiologist. Circulation 2002105 546-549.

17 Yudkin JS, Stehouwer CDA, Emeis JJ \& Coppack SW. C-rective protein in healthy subjects: associations with obesity, insulin resistance, and endothelial dysfunction. A potential role for cytokines originating from adipose tissue. Arteriosclerosis Thrombosis and Vascular Biology 199919 972-978.

18 Festa A, D’Agostino R, Howard G, Mykkanen L, Tracy R \& Haffner S. Chronic subclinical inflammation as part of insulin resistance syndrome: the insulin resistance atherosclerosis study (IRAS). Circulation $200010242-47$.

19 Choi KM, Lee KW, Seo JA, Oh JH, Kim SG, Kim NH \& Baik SH. Comparison of serum concentrations of C-reactive protein, TNFalpha, interleukin 6 between elderly Korean women with normal and impaired glucose tolerance. Diabetes Research and Clinical Practice 200464 99-106.

20 Dunaif A, Scott D, Finegood D, Quintana B \& Whitcomb R. The insulin sensitizing agent troglitazone improves metabolic and reproductive abnormalities in the polycystic ovary syndrome. Journal of Clinical Endocrinology and Metabolism 1996 $813299-3306$.

21 Ehrmann DA, Schneider DJ, Sobel BE, Cavaghan MK, Imperial J, Rosenfield RL \& Polonsky KS. Troglitazone improves defects in insulin action, insulin secretion, ovarian steroidogenesis, and fibrinolysis in women with polycystic ovary syndrome. Journal of Clinical Endocrinology and Metabolism 199782 2108-2116.

22 Azziz R, Ehrmann D, Legro R, Whitcomb RW, Hanley R, Gmerek Fereshetian A, O'Keefe M \& Ghazzi MN. PCOS/Troglitazone Study Group: troglitazone improves ovulation and hirsutism in polycystic ovary syndrome: a multicenter, double blind, placebo-controlled trial. Journal of Clinical Endocrinology and Metabolism 200186 1626-1632.

23 Paradisi G, Steinberg HO, Shepard MK, Hook G \& Baron AD. Troglitazone therapy improves endothelial dysfunction to near normal levels in women with polycystic ovary syndrome. Journal of Clinical Endocrinology and Metabolism $2003 \mathbf{8 8} 576-580$.

24 Pistrosch F, Passauer J, Fisher S, Fuecker K, Hanefeld M \& Gross P. In type 2 diabetes, rosiglitazone therapy for insulin resistance ameliorates endothelial dysfunction independent of glucose control. Diabetes Care 200427 484-490.

25 Sidhu JS, Cowan D \& Kaski JC. The effects of rosiglitazone, a peroxisome proliferator activated receptor-gamma agonist, on markers of endothelial cell activation, $\mathrm{C}$ reactive protein, and fibrinogen levels in non-diabetic coronary artery disease patients. Journal of American Collage of Cardiology 200319 1757-1763.

26 Wang TD, Chen WJ, Lin JW, Chen MF \& Lee YT. Effects of rosiglitazone on endothelial dysfunction, C-reactive protein and components of the metabolic syndrome in nondiabetic patients with metabolic syndrome. American Journal of Cardiology 2004 $3362-365$.

27 The Rotterdam ESHRE/ASRM-Sponsored PCOS Consensus Workshop Group, Revised 2003 consensus on diagnostic criteria and long-term health risks related to polycystic ovary syndrome. Fertility and Sterility 200481 19-24.

28 Ferriman D \& Gallwey JD. Clinical assessment of body hair growth in women. Journal of Clinical Endocrinology and Metabolism 1962 21 1440-1447.

29 Matthews DR, Hosker JP, Rudenski AS, Naylor BA, Trecher DF \& Turner DC. Homeostasis model assessment: insulin resistance and $\beta$-cell function from fasting plasma glucose and insulin concentrations in man. Diabetologia $1985 \mathbf{2 8} 412-419$.

30 Katz A, Nambi SS \& Mather K. Quantitative insulin sensitivity check index: a simple, accurate method for assessing insulin sensitivity in humans. Journal of Clinical Endocrinology and Metabolism $2000852402-2410$.

31 Coretti MC, Anderson TJ, Benjamin EJ, Celermajer D, Charbonneau F, Creager MA, Deanfield J, Drexler H, GerhardHerman M, Herrington D, Vallance P, Vita J \& Vogel R. Guidelines for the ultrasound assessment of endothelium-dependent flowmediated vasodilation of the brachial artery. A Report of the International Brachial Artery Reactivity Task Force. Journal of American Collage of Cardiology 200239 257-265.

32 Kushner I. Regulation of acute phase response by cytokines. Perspectives in Biology and Medicine 199336 611-622.

33 Shimokawa H. Primary endothelial dysfunction: atherosclerosis. Journal of Molecular and Cellular Cardiology 199931 23-27.

34 Anderson TJ. Assessment and treatment of endothelial dysfunction in humans. Journal of the American College of Cardiologists 199934 631-638.

35 Panza JA. Endothelial dysfunction in essential hypertension. Clinical Cardiology 199720 26-33.

36 Creager MA, Cooke JP, Mendelsohn ME, Gallagher SJ, Coleman SM, Loscalzo J \& Dzau VJ. Impaired vasodilation of fore- 
arm resistance vessels in hypercholesterolemic humans. Journal of Clinical Investigations $1990 \mathbf{8 6} 228-234$.

37 Arcaro G, Zamboni M, Rossi L, Turcato E, Covi G, Armellini F, Basella $\mathrm{O} \&$ Lechi A. Body fat distribution predicts the degree of endothelial dysfunction in uncomplicated obesity. International Journal of Obesity 199923 936-942.

38 Steinberg HO, Chaker H, Leaming R, Johnson A, Brechtel G \& Boron AD. Obesity/insulin resistance is associated with endothelial dysfunction. Implications for the syndrome of insulin resistance. Journal of Clinical Investigations $1996112601-2610$.

39 Dunaif A. Insulin resistance and polycystic ovary syndrome: mechanism and implications for pathogenesis. Endocrine Reviews $199718774-780$.

40 Richardson MR. Current perspectives in polycystic ovary syndrome. American Family Physician 200368 697-704.

41 Nestler JE, Powers LP \& Matt DW. A direct effect of hyperinsulinemia on serum sex hormone-binding globulin levels in obese women with polycystic ovary syndrome. Journal of Clinical Endocrinology and Metabolism 198968 1027-1032.

42 Dunaif A, Segal KR, Futterweit W \& Dobrjansky A. Profound peripheral insulin resistance independent of obesity in polycystic ovary syndrome. Diabetes 198938 1165-1174.

43 Vrbikova J, Bendlova B, Hill M, Vankova M, Vondra K \& Starka L. Insulin sensitivity and $\beta$-cell function in women with polycystic ovary syndrome. Diabetes Care 200225 1217-1222.

44 Paradisi G, Steinberg HO, Hempfling A, Cronin J, Hook G, Shepard MK \& Baron AD. Polycystic ovary syndrome is associated with endothelial dysfunction. Circulation 1999103 1410-1415.

45 Christopher J, Kelly JG, Speirs A, Gwyn WG, Petrie JR, Lyall H \& Connell JMC. Altered vascular function in young women with polycystic ovary syndrome. Journal of Clinical Endocrinology and Metabolism $200287742-746$.

46 Ridker PM, Stampfer MJ \& Rifai N. Novel risk factors for systemic atherosclerosis: a comparison of C-reactive protein, fibrinogen, homocysteine, lipoprotein(a) and standard cholesterol screening as predictors of peripheral arterial disease. Journal of the American Medical Association $20012852481-2485$.
47 Ridker PM. High-sensitivity C-reactive protein: potential adjunct for global risk assessment in primary prevention of cardiovascular disease. Circulation 2001103 1813-1818.

48 Pasceri V, Cheng JS, Willerson JT, Yeh ET \& Chang J. Modulation of C-reactive protein-mediated monocyte chemoattractant protein-1 induction in human endothelial cells by anti-atherosclerosis drugs. Circulation $2001 \mathbf{1 0 3} 2531-2534$.

49 Kelly CJJ, Lyall H, Petrie JR, Gould GW, Connell CMJ \& Sattar N. Low grade chronic inflammation in women with polycystic ovary syndrome. Journal of Clinical Endocrinology and Metabolism $2001862453-2455$.

50 Sepilian V \& Nagamani M. Effects of rosiglitazone in obese women with polycystic ovary syndrome and severe insulin resistance. Journal of Clinical Endocrinology and Metabolism 200590 60-65.

51 Baillargeon J, Jakubowicz DJ, Iuomo MJ, Jakubowicz S \& Nestler JE. Effects of metformin and rosiglitazone, alone and in combination, in nonobese women with polycystic ovary syndrome and normal indices of insulin sensitivity. Fertility and Sterility $2004 \mathbf{8 2}$ 893-902.

52 Vinik AI, Stansberry KB \& Barlow PM. Rosiglitazone treatment increases nitric oxide production in human peripheral skin. Journal of Diabetes and its Complications 200317 279-285.

53 Mohanty P, Aljada A, Ghanim H, Hofmeyer D, Tripahy D, Syed T, Al-Haddad W, Dhindsa S \& Dandona P. Evidence for a potent antiinflammatory effect of rosiglitazone. Journal of Clinical Endocrinology and Metabolism $2004892728-2735$.

54 Haffner SM, Greenberg AS, Weston WM, Chen H, Williams K \& Freed MI. Effect of rosiglitazone treatment on nontraditional markers of cardiovascular disease in patients with type 2 diabetes mellitus. Circulation 2002106 679-684.

Received 1 February 2005

Accepted 13 April 2005 